Article

\title{
Assessment of Residual Elastic Properties of a Damaged Composite Plate with Combined Damage Index and Finite Element Methods
}

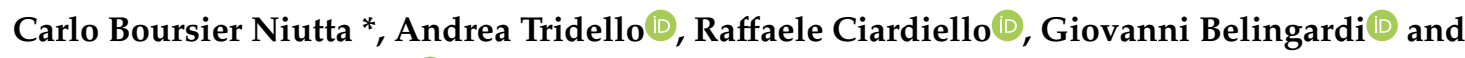 \\ Davide Salvatore Paolino $(\mathbb{D}$ \\ Department of Mechanical and Aerospace Engineering, Politecnico di Torino, 10129 Turin, Italy; \\ andrea.tridello@polito.it (A.T.); raffaele.ciardiello@polito.it (R.C.); giovanni.belingardi@polito.it (G.B.); \\ davide.paolino@polito.it (D.S.P.) \\ * Correspondence: carlo.boursier@polito.it
}

Received: 29 May 2019; Accepted: 21 June 2019; Published: 25 June 2019

\begin{abstract}
In structural component applications the use of composite materials is increasing thanks to their optimal mechanical characteristics. However, the complexity of the damage evolution in composite materials significantly limits their widespread diffusion. Non-destructive tests are thus becoming ever more important. The detecting Damage Index $\left(D I_{d}\right)$ technique has been recently brought in the realm of the non-destructive characterization tests for components made of composite material. In contrast to other techniques, this methodology allows to quantitatively assess local residual properties. In this paper, the $D I_{d}$ technique is adopted in combination with the finite element method. The mechanical response of two composite plates (an 8-layer twill fabric carbon/epoxy) subjected to four-point bending test is firstly used to tune a finite element model of the laminate. Then, an undamaged laminate of the same composite material is progressively damaged through repeated four-point bending tests. Local residual elastic properties are mapped on the plate through the $D I_{d}$ technique. A continuous polynomial curve has been considered to account for the variation of the elastic modulus in the finite element model. The resulting force-displacement curve of the numerical analysis is compared to experimental data of damaged plate, resulting in very good agreement. The combination of the experimental activity and the numerical finite element analysis points out the accuracy of the $D I_{d}$ methodology in assessing local residual elastic properties of composite materials.
\end{abstract}

Keywords: non-destructive tests; damage assessment; residual properties; Finite Element Method; Damage Index

\section{Introduction}

Composite materials are increasingly adopted in structural-mechanical applications thanks to their optimal characteristics in terms of light weight, mechanical strength and stiffness, corrosion resistance, energy absorption capacity, and noise attenuation. However, many factors limit their widespread diffusion, such as the high cost of raw materials or requirements for high production volumes. Among the others, the complexity of the damage evolution plays a key role in their limitation, especially for structural component applications. Several interacting failure modes are typical of composite materials and a progressive and rapid decrement of the mechanical properties can be observed [1].

In this regard, methodologies for assessing damage level and predicting the residual structural strength of composite materials are becoming increasingly important. Many techniques have been developed for non-destructively assessing the structural health state of composite components [2]. 
Non-destructive tests (NDTs) are commonly adopted for quality assessment of a manufacturing process or for damage evaluation in structures during service. Most of the techniques intend to provide information on internal damages, in terms of size, shape, location, and orientation. Microscopy (based on atomic force, optic system, and scanning and transmission electron) [3], X-ray micro-CT (micro-computed tomography) [3,4], and infrared thermography $[3,5]$ are among the most used for composite applications. However, the qualitative information provided by these techniques can be exploited by designers with difficulty. Further, methods based on ultrasounds and vibrational analysis are usually adopted for globally analyzing the dynamic response of a structure [6]. In particular, the use of the Impulse Excitation Technique (IET), which is regulated by ASTM Standards for metallic materials $[7,8]$, has been recently extended to composite specimens [9]. However, local damages and defects are still revealed with difficulty in real-world structures since the presence of anomalies is mitigated by the global response of the system. Therefore, as pointed out in [10], current methodologies do not allow a direct evaluation of the local residual properties.

In this paper, the detecting Damage Index $\left(D I_{d}\right)$ technique was adopted for assessing the local residual elastic properties of a composite laminate. This methodology was brought in the realm of the non-destructive characterization for components made of composite material by Belingardi et al. in [11-15]. The $D I_{d}$ technique consists of two sets of experimental tests: a preliminary characterization activity which is performed to identify the correlation between residual elastic properties of the material and the $D I_{d}$ parameter and the proper non-destructive test to estimate the local residual elastic properties of the investigated component from the $D I_{d}$. In [15], the methodology was demonstrated to properly predict residual elastic properties of a series of laminate plates damaged through impacts at different energy levels. Tensile tests on specimens cut from laminate plates allowed validation of results predicted with the $D I_{d}$. Here, the finite element method was adopted to validate elastic properties assessed with the $D I_{d}$. The combination of non-destructive tests and finite element method is typical in damage assessment, particularly in vibration-based techniques [16]. Various approaches are available to numerically model multi-layered composite materials and their multiple failure modes $[17,18]$. In particular, the use of cohesive elements disposed between layers of shells currently represents one of the most adopted solutions [19-21]. Thanks to their specific formulation, cohesive elements allows to simulate damages due to delamination without significantly affecting computational cost.

In this paper, the $D I_{d}$ technique is thus used in combination with the finite element method. An 8 layers twill fabric carbon/epoxy composite laminate is firstly damaged through repeated four-point bending tests. Then, the $D I_{d}$ technique is adopted to map the residual elastic properties on the damaged plate. The variation of the Young's modulus is accounted in the finite element model of the laminate subjected to bending test through a continuous polynomial curve. The resulting experimental and numerical force-displacement curves are then compared to validate the proposed methodology. The combination of the experimental activity and the numerical finite element analysis points out the accuracy and the effectiveness of the $D I_{d}$ methodology in predicting the local residual elastic properties of damaged composite components.

\section{Materials and Methods}

In this section, investigated materials are firstly detailed. Then experimental and numerical methods are presented.

\subsection{Materials}

Experimental tests are performed on a structural composite laminate specifically developed for automotive application. The composite material is the same adopted by Tridello et al. in their investigations [15] and previously in [9]. It consists of a matrix made of epoxy resin reinforced by eight layers of twill woven carbon fabric. The first layer is a $380 \mathrm{gsm}$ fabric with $0.45 \mathrm{~mm}$ thickness and an $800 \mathrm{gsm}$ fabric with $0.88 \mathrm{~mm}$ thickness is used in the remaining seven layers. The stacking sequence is $[0 / 90]_{8}$. In the numerical analysis, the laminate is modeled as symmetric, with 8 layers 
of thickness $0.88 \mathrm{~mm}$, oriented according to the stacking sequence. A total thickness of $7.04 \mathrm{~mm}$ is thus obtained. Even though the first layer is different from the other seven, this difference can be neglected when considering the global mechanical behavior of the laminate. The measured elastic properties (Young's modulus, shear modulus, and Poisson's ratio) are reported in Table 1, as assessed in [9]. Given the symmetry of the woven fabric, the mechanical properties and the Poisson's ratios in the in-plane direction 1 and 2 are the same (i.e. $E_{1}=E_{2}, v_{12}=v_{21}$ ).

Table 1. Material properties.

\begin{tabular}{cc}
\hline Property & Value \\
\hline Density & $1.4510^{3} \mathrm{~g} / \mathrm{cm}^{3}$ \\
Young's modulus in longitudinal and transverse direction $\left(E_{1}=E_{2}\right)$ & $54 \mathrm{GPa}$ \\
Shear modulus $\left(G_{12}\right)$ & $3.5 \mathrm{GPa}$ \\
Poisson's ratio $\left(v_{12}=v_{21}\right)$ & 0.08 \\
\hline
\end{tabular}

\subsection{Experimental Tests}

Four-point bending tests are performed on two different composite plates until complete failure occurs. The elastic field of the composite plates is thus identified. The tests are performed on a servohydraulic testing machine (Instron 8801). In agreement with the recommendations of ASTM standard D6272 for four-point bending test of reinforced plastics [22], the load span is one third of the support span. As shown in Figure 1, the loading noses are $50 \mathrm{~mm}$ distant. The support noses are consequently placed at a distance of $150 \mathrm{~mm}$. Figure 1a shows the experimental setup. The resulting experimental curves of force with respect to displacement are exploited to tune a finite element model of the laminate subjected to four-point bending test, which is shown in Figure 1b.

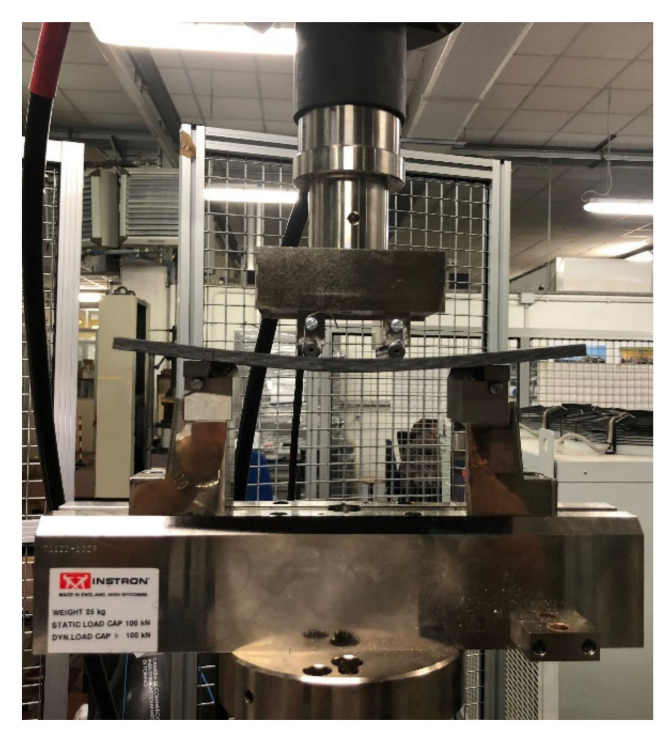

(a)
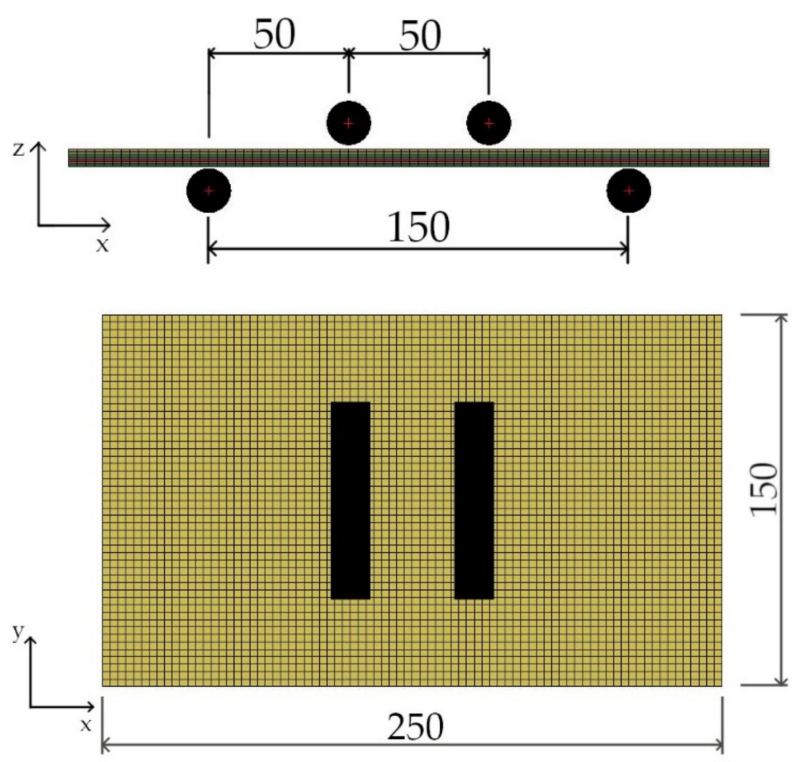

(b)

Figure 1. Four-point bending test: (a) experimental setup; (b) numerical model.

The same experimental setup is adopted to progressively damage an undamaged composite plate. The plate is loaded until incipient failure occurs and then unloaded. The repeated four-point bending tests progressively reduce plate stiffness. A significant damage level is thus induced.

In order to assess the residual Young's modulus of the damaged plate, the detecting Damage Index $\left(D I_{d}\right)$ technique is adopted. The $D I_{d}$ methodology consists of two sets of experimental tests. Firstly, a preliminary characterization activity is performed, which allows to identify the correlation between 
residual elastic properties and the $D I_{d}$ parameter. Then, the investigated component undergoes the non-destructive test in order to estimate the local residual elastic properties from the $D I_{d}$.

In particular, the methodology is based on two sets of impact tests: the first intends to damage the material at increasing impact energy levels and allows to evaluate the so-called threshold energy $\varepsilon_{t h}$. The threshold energy is defined as the impact energy at which the reduction of local elastic properties is less than $5 \%$. An impact at the threshold energy can be considered as non-destructive for the material. The second set of impacts is performed at the threshold energy and allows to evaluate the residual elastic properties.

In this work, the first series of impacts were performed on a set of plates made of the same composite fabric. The impact tests were carried out using a free-fall drop dart testing machine (CEAST 9350 FRACTOVIS PLUS). Clamping boundary conditions were realized through a mechanical clamping system which applies an almost uniform pressure on the clamped area. A circular unclamped region of diameter $76 \mathrm{~mm}$ was considered for the tests, in agreement with the recommendations of ASTM standard D5628 [23]. The impact energy was defined by varying the impactor mass for a given impact velocity. The impact velocity, which was controlled by the drop height of the dart, was measured in each test with an optoelectronic device. A piezoelectric load cell, mounted in proximity of the tip of the impact dart, acquired the force signal at a sample rate of $1 \mathrm{MHz}$.

The second series of impacts, which are carried out at the threshold energy, is performed on the plate previously damaged with the repeated four-point bending tests. In particular, damaged plate is impacted along the middle line. Moving along the longitudinal direction of the plate ( $x$ direction of Figure 1), the impacts are located in correspondence of the external noses, in the regions where bending moment is linearly increasing, in correspondence of the internal noses and finally in the middle of the load span. Seven impacts are applied in total to the plate, as shown in Figure 2. The corresponding residual Young's modulus is calculated from the $D I_{d}$ parameter, which is defined as

$$
D I_{d}=\frac{\varepsilon_{a}}{\varepsilon_{t h}} \cdot \frac{s_{M A X}}{s_{Q S}}
$$

where $\varepsilon_{a}$ is the absorbed energy, $\varepsilon_{t h}$ the threshold energy, $s_{M A X}$ the maximum displacement and $s_{Q S}$ the displacement obtained in quasi static perforation test. The $s_{M A X}$ and $\varepsilon_{a}$ values are computed through numerical integrations of the load signal acquired during the impact test.

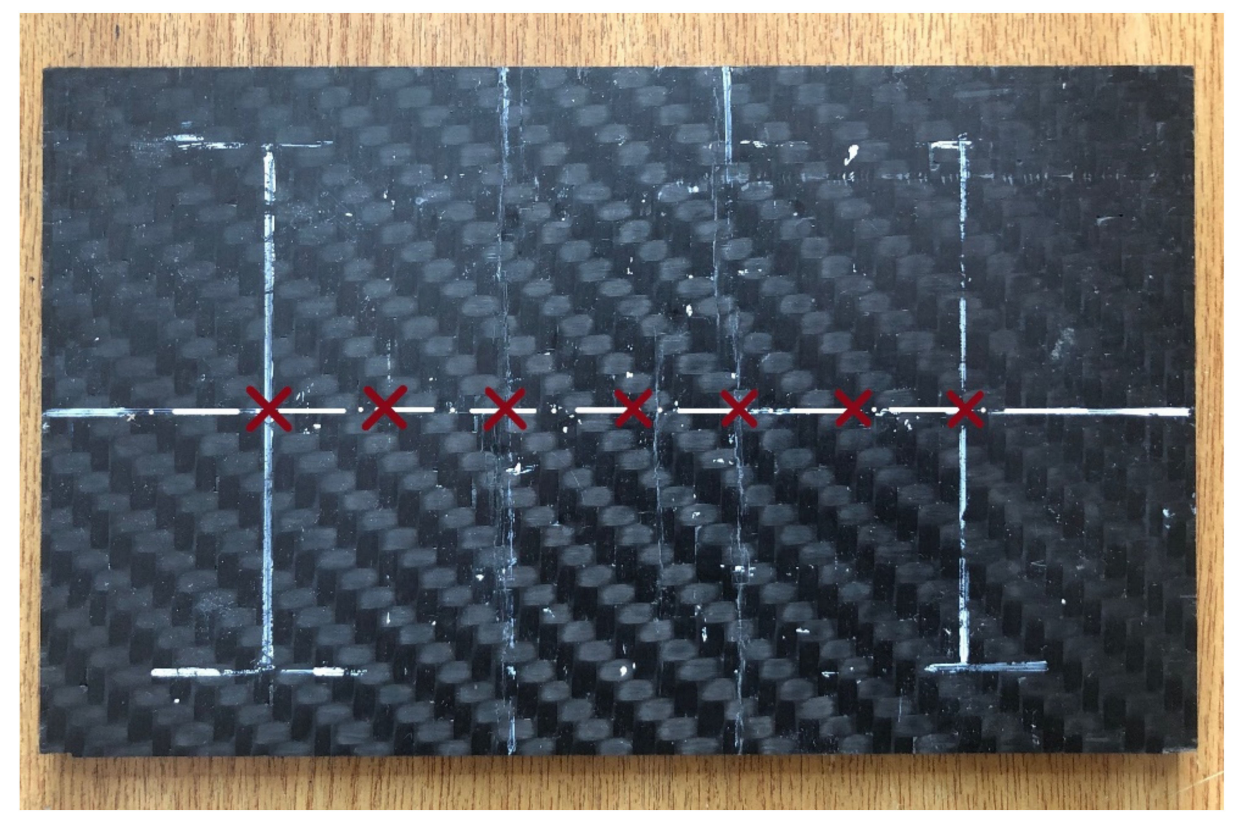

Figure 2. Location of impacts for $D I_{d}$ analysis. 


\subsection{Numerical Model}

A structural-mechanical model of the four-point bending test was simulated using the commercial software LS-DYNA. The 8 layers of the woven fabric composite were modeled with Belytschko-Tsai 4-node shell elements, with one integration point through the thickness. For each layer of the composite, a layer of shell elements was considered. Solid elements of cohesive material were placed between the shell layers, in order to represent the resin among layers. Solid elements of cohesive consider a specific formulation of LS-DYNA, which allows the transfer of moments to the shells. In particular, nodes of solid elements coincide with those of the shells. The material card *MAT_LAMINATED_COMPOSITE_FABRIC, which is specific for fabric composite, was adopted for the composite layers. This material model assumes a slightly modified version of Hashin criteria for failure [18]. However, in this paper, the focus is on the elastic field, and parameters related to the post-failure behavior of the material will not be addressed. The cohesive material model *MAT_COHESIVE_TH, with properties of the resin, was assigned to the cohesive elements. Finally, the span noses were modeled as rigid cylinder walls. The upper cylinders, shown in Figure 1b, move downwards with a prescribed motion law. The upper and lower cylinders were placed at the initial distance of $1 \mathrm{~mm}$ from the laminate in order to avoid penetrations and numerical instabilities. Contact between laminate and cylinders was based on a penalty formulation with a penalty factor which adaptively guarantees the numerical stability [24]. This formulation accounts for thickness offsets of shells.

The accuracy of the numerical model was firstly verified with respect to the four point-bending tests of the two composite plates. Then, the same numerical model was used in comparison with the experimental results of the progressively damaged plate. In this case, the structural-mechanical model had to account for the variation of Young's modulus on the plate, as pointed out through the $D I_{d}$ technique.

The residual elastic properties of the damaged plate are mapped through the $D I_{d}$ technique. The Young's modulus varies along the x-direction according to the location of measurement, with the damage mostly located in correspondence of internal rolls. It seems reasonable to assume that the Young's modulus varies continuously along the longitudinal direction. Here, a polynomial curve is considered for each region included between two consecutive measure locations.

For each polynomial curve, the two Young's moduli at the extremities, $E_{k}$ and $E_{k+1}$, with $\mathrm{k}$ the considered location, are known.

In addition, the two derivatives at the extremities can be estimated. In order to evaluate the derivates, the difference quotient for each region has been calculated as

$$
\frac{\Delta E_{k}}{\Delta x_{k}}=\frac{E_{k}-E_{k-1}}{x_{k}-x_{k-1}}
$$

in accordance with its definition.

The difference quotient is a measure of the average rate of change of the function, here the Young's modulus, over the interval $\Delta x_{k}$. By considering two consecutive difference quotients, $\frac{\Delta E_{k}}{\Delta x_{k}}$ and $\frac{\Delta E_{k+1}}{\Delta x_{k+1}}$, the derivative at the $\mathrm{k}$-th location has been estimated as follows:

1. when the product $\frac{\Delta E_{k}}{\Delta x_{k}} \cdot \frac{\Delta E_{k+1}}{\Delta x_{k+1}}$ returns a negative value, the derivate at the $\mathrm{k}$-th location is assumed equal to zero. This can be justified by considering that a change in the sign of the difference quotient implies a change in the derivative of the function, as well;

2. when the sign of the product $\frac{\Delta E_{k}}{\Delta x_{k}} \cdot \frac{\Delta E_{k+1}}{\Delta x_{k+1}}$ is positive, the derivate at the k-th location is assumed equal to average value $\frac{\frac{\Delta E_{k}}{\Delta x_{k}}+\frac{\Delta E_{k+1}}{\Delta x_{k+1}}}{2}$;

3. the derivates at the extremities, $k=1$ and $k=7$, are assumed equal to zero. 
As the two Young's moduli and the derivatives are known at the extremities, a third-order polynomial curve can be constructed for each interval included between two consecutive impact locations. This approach guarantees the continuity of the Young's modulus with respect to the x-coordinate.

In the structural-mechanical model, this variation was accounted by longitudinally dividing the layers of shells into parts. Each part consisted of one row of shell elements and is identified by its x-coordinate. In particular, each part could be longitudinally localized in correspondence of the middle of the element size. In this work, a mesh of $3 \mathrm{~mm}$ was adopted. According to the location of the part in the $x$ direction, the corresponding third-order polynomial curve could be identified. The Young's modulus was consequently calculated. A material card was defined for each part with the corresponding elastic properties.

\section{Results}

In this Section, the experimental and numerical results are presented. In Section 3.1, experimental and numerical data related to the four-point bending tests are compared. In Section 3.2, results of the progressive damaging of the composite plate through the repeated four-point bending tests are shown. Residual elastic properties are thus estimated through the $D I_{d}$ methodology. The Young's modulus is mapped with respect to the longitudinal coordinate and the third-order polynomial curves were constructed as previously described. Finally, in Section 3.3, the proposed approach is validated by comparing the experimental results of the progressively damaged plate with the numerical model which accounts for the residual properties.

\subsection{Four-Point Bending Test: Experimental Results and Numerical Model Tuning}

Results of the four-point bending tests are here presented. Two undamaged composite plates have been tested until almost complete failure occurred. In particular, the upper layers, which are subjected to compressive loads, showed the most significant failures. Intralaminar cracks propagate in correspondence of the inner span noses. The numerical model is analyzed only in the elastic field and it results in good agreement with the experimental data, as shown in Figure 3. Displacement of the numerical analysis has been evaluated on the rigid cylinders. However, as described in Section 2.2, the rigid wall cylinders are initially distanced from the composite laminate. Consequently, the initial path of the force-displacement curve is characterized by the growing contact between laminate and cylinders, which is not significant for the purpose. The numerical force-displacement curve has been thus shifted leftwards and results of the numerical analysis are reported from $1 \mathrm{~mm}$ of displacement.

Figure 4 shows a magnification of the cracks, obtained through an optical microscope. The complete failure can be appreciated for the upper four layers. 


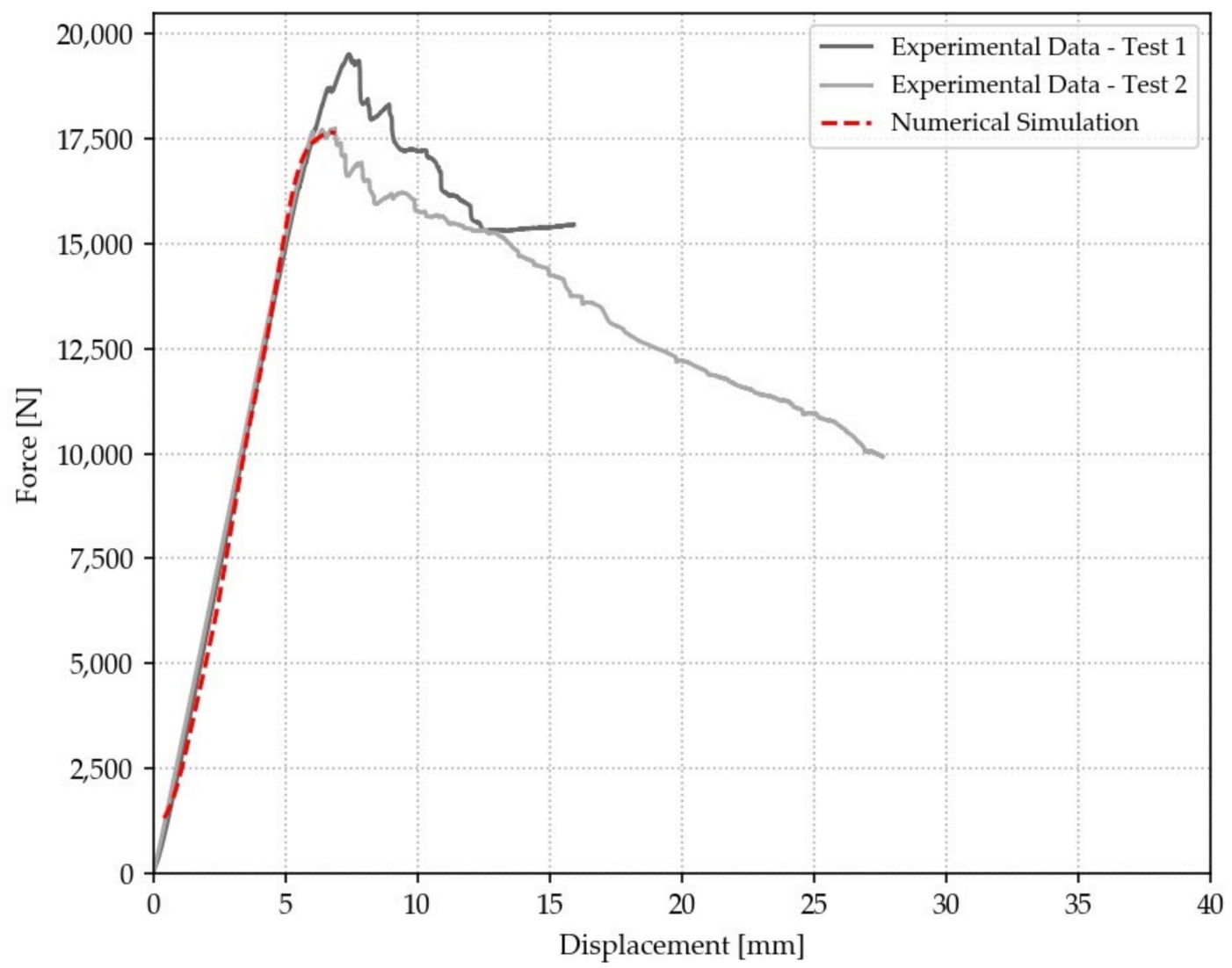

Figure 3. Experimental and numerical results of the four-point bending tests.

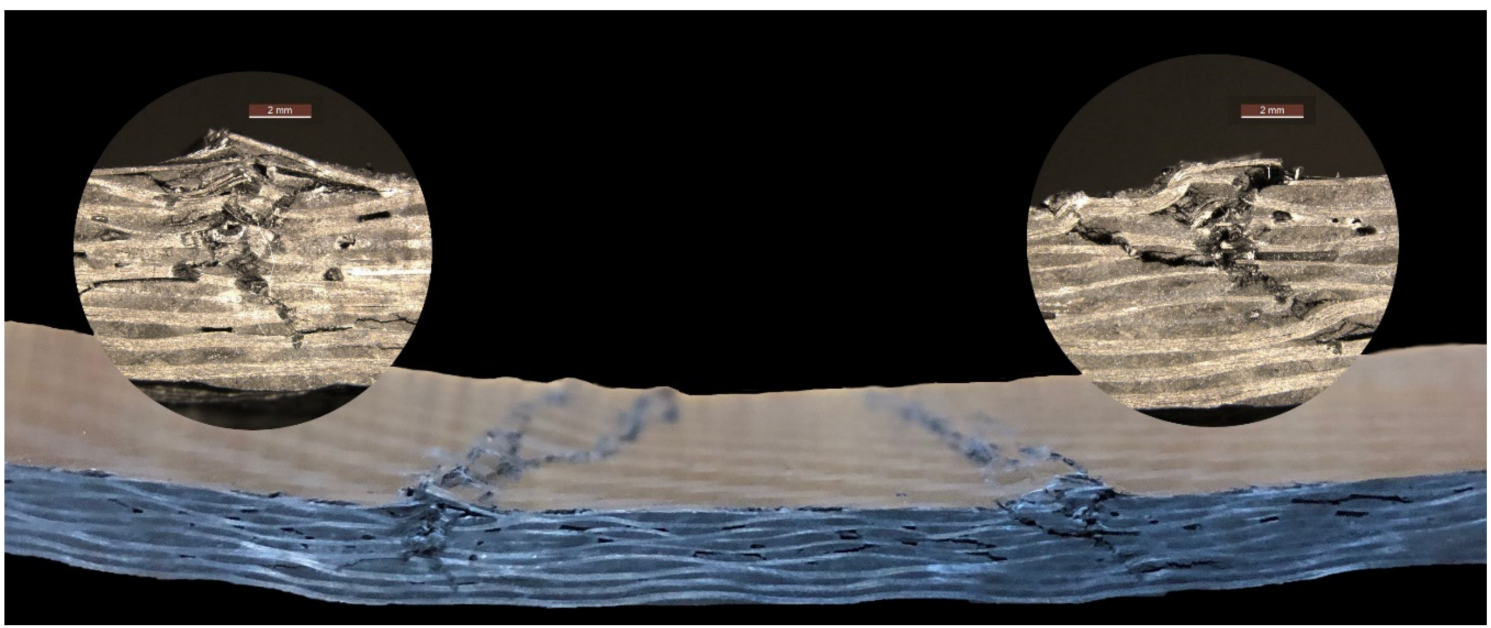

Figure 4. Magnification of intralaminar cracks: failure of the upper four layers.

\subsection{Progressive Damaging and Assessment of Residual Elastic Properties through the $D I_{d}$ Technique}

An undamaged composite is subjected to repeated four-point bending tests. Load is increased until incipient failure occurs and then decreased. Achieved failure is not complete, as the load-carrying capacity of the composite laminate is still significant. In total, five repetitions are performed, as shown in Figure 5, where the resulting force-displacement curves are numbered as Test n.1, Test n.2, Test n.3, Test n.4, and Test n.5. As the damage increases, the plate stiffness progressively decreases. Loading and unloading cycles are performed until the residual stiffness of the composite plate is about $40 \%$ of the original value. This is shown with the Test n.6 (damaged specimen curve), which is performed only in the elastic field. A significant damage level is thus induced through the five tests. 


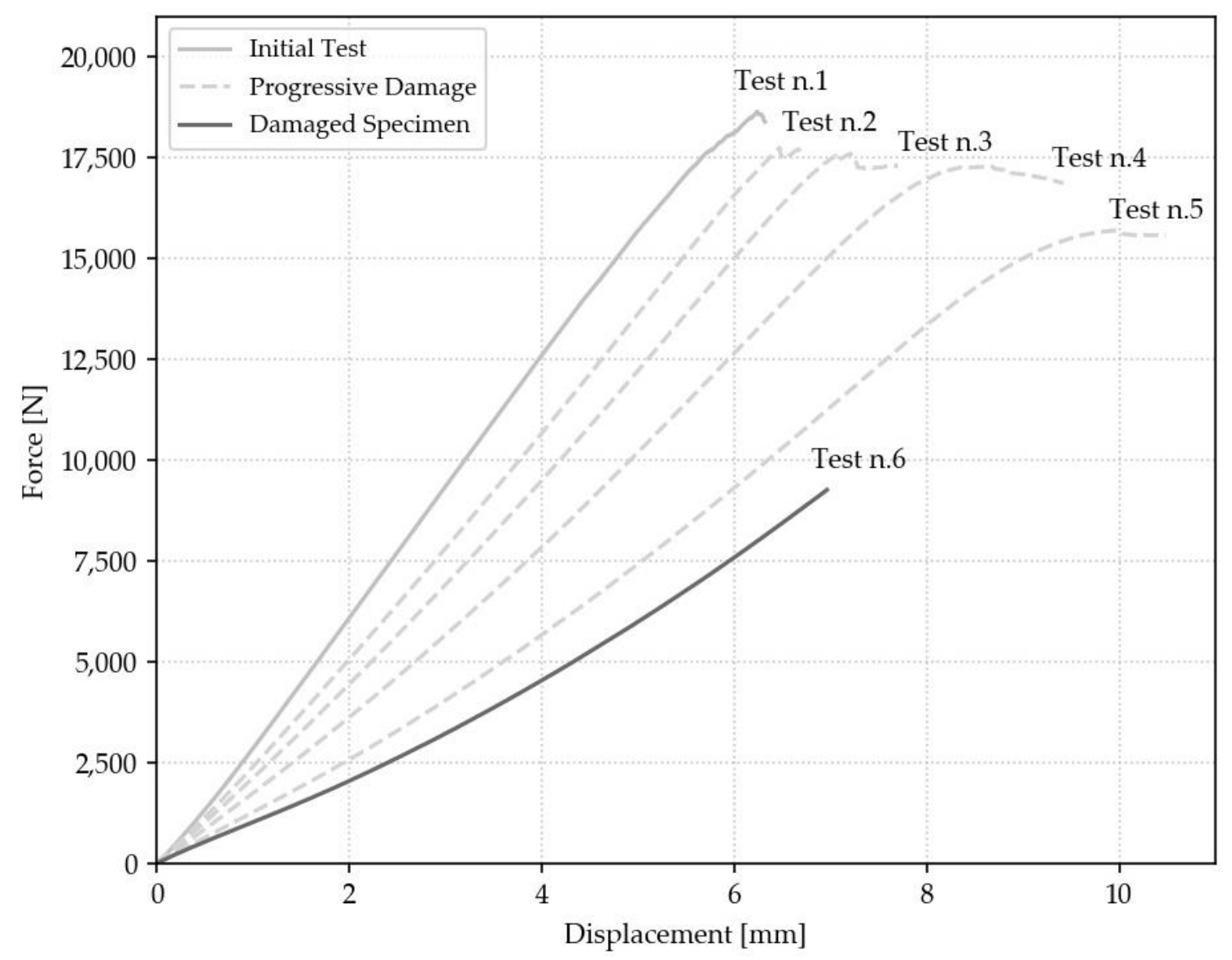

Figure 5. Progressive damaging of composite plate through four-point bending tests.

The $D I_{d}$ technique is then adopted to estimate the residual elastic properties. Preliminary characterization tests allowed to evaluate the threshold energy $\varepsilon_{t h}$, which is in this case equal to $5 \mathrm{~J}$. Details of the preliminary tests are not discussed here. A complete description of the procedure can be found in [15].

Figure 6 shows the resulting correlation between the residual elastic properties of the analyzed composite and the $D I_{d}$ parameter. The dotted line is thus adopted to evaluate the residual elastic properties in the progressively damaged plate. Cross markers correspond to the preliminary characterization tests. 


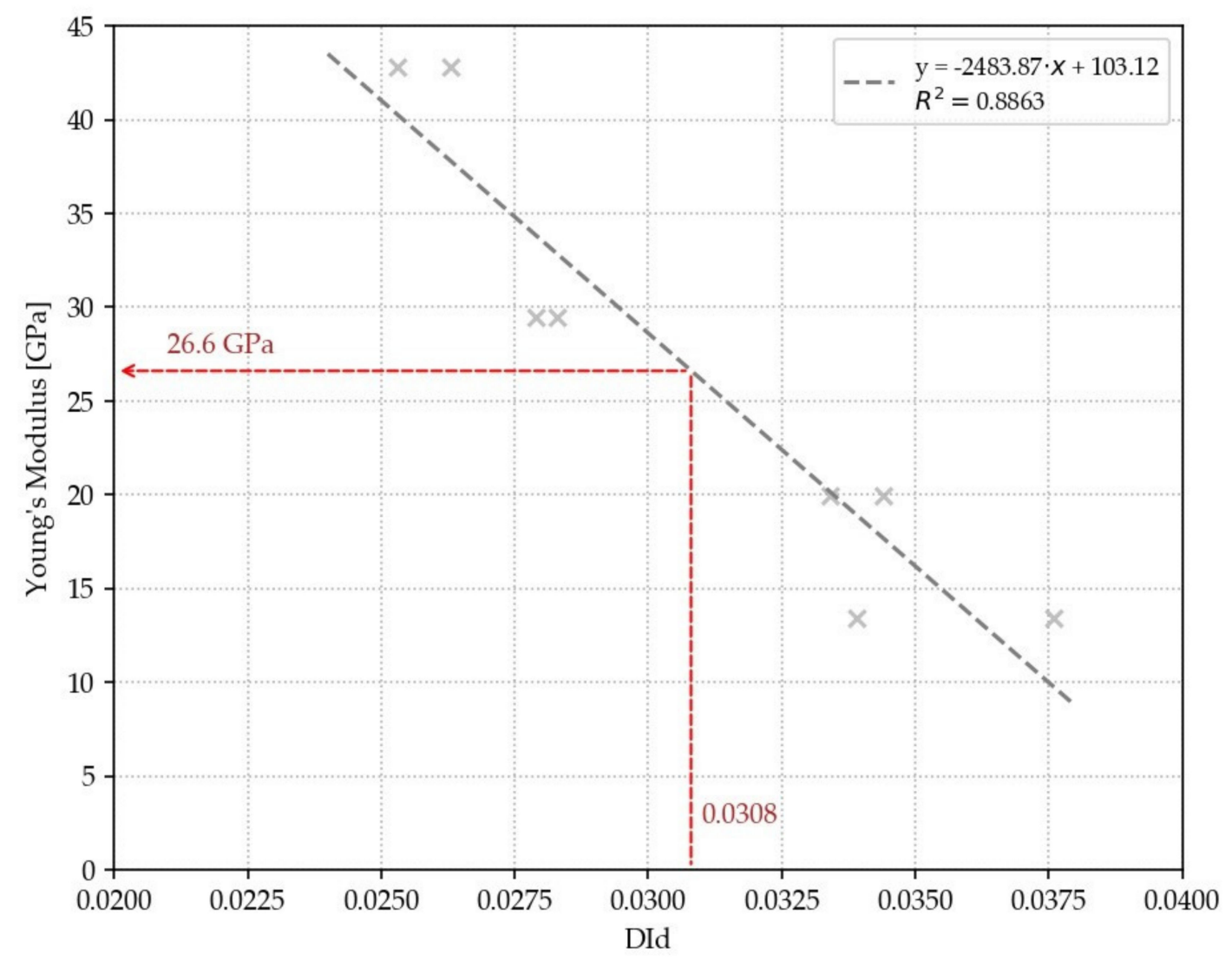

Figure 6. Residual Young's modulus evaluation with $D I_{d}$.

As reported in Figure 2, the elastic properties have been estimated at seven locations along the middle line of the composite plate. Table 2 reports the measured $D I_{d}$ values and the corresponding Young's moduli, as well as the locations in $\mathrm{x}$ direction of the impacts.

Table 2. Residual Young's moduli and corresponding $D I_{d}$ values at specific locations.

\begin{tabular}{ccc}
\hline X-Coordinate $[\mathrm{mm}]$ & $\boldsymbol{D I}_{\boldsymbol{d}}$ & $\boldsymbol{E}_{\text {res }}[\mathrm{GPa}]$ \\
\hline 50 & 0.0283 & 32.8 \\
75 & 0.0297 & 29.5 \\
100 & 0.036 & 13.7 \\
125 & 0.0308 & 26.6 \\
150 & 0.036 & 13.7 \\
175 & 0.0297 & 29.5 \\
200 & 0.0283 & 32.8 \\
\hline
\end{tabular}

In correspondence of the external noses, whereas the bending moment is null in the four-point bending test and no damage should be present, the elastic modulus is equal to $32.8 \mathrm{GPa}$, which is significantly different from the original value of $54 \mathrm{GPa}$. This can be justified by taking into account that the circular unclamped region considered for the $D I_{d}$ test has a diameter of $76 \mathrm{~mm}$. Consequently, elastic properties are assessed as an average of the unclamped region.

Further, as clamping boundary conditions have to be realized all around the laminate, the diameter of $76 \mathrm{~mm}$ limits the region where the residual properties can be measured through the $D I_{d}$. Moving outwards from the external noses, only few measurements of the residual properties can be realized and results would be affected by the damaged portion of the plate. On the basis of these considerations, we can assume that, at a distance of $38 \mathrm{~mm}$ from the external noses (half of the diameter), the damaged 
portion of the plate would not affect an ideal measurement through the $D I_{d}$. The Young's modulus is thus here considered equal to the original value of $54 \mathrm{GPa}$.

From the seven measurements of the Young's modulus and considering the two assumed external values, eight third-order polynomial curves can be constructed, according to the approach described in Section 2.2. As a consequence, a continuous variation of the Young's modulus with respect to the x-coordinate is obtained. This result is shown in Figure 7, where measured Young's moduli are marked with red squares and assumed values with black thin diamonds. The continuous curve is adopted in the numerical model to account for the variation of the Young's modulus. Layers of shells have been longitudinally divided into parts, which are represented with different colors in Figure 8. The continuous curve allows to evaluate the Young's modulus in correspondence of the x-coordinate of each part. A material card has been defined for each part with the corresponding elastic properties. Cross markers of Figure 7 represent the Young's moduli assigned to each material card.

Moving outwards from the external noses, the elastic modulus increases from 32.8 to $54 \mathrm{GPa}$ and then is assumed constant. It should be noted that, as the bending moment is here null, the mechanical behavior of the numerical model will not be affected by the value of the elastic modulus estimated for this region.

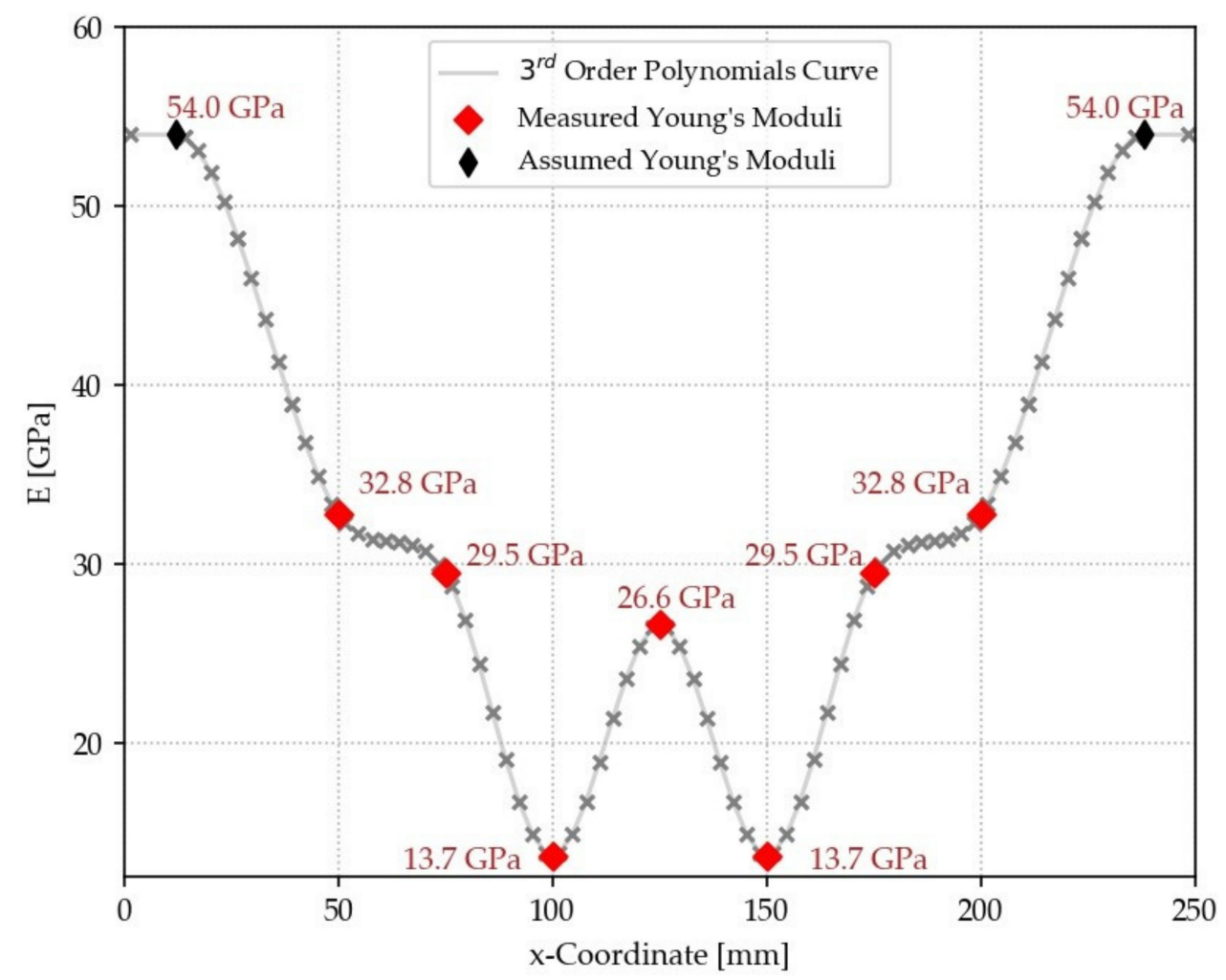

Figure 7. Variation of residual Young's modulus with respect to the x-coordinate. 


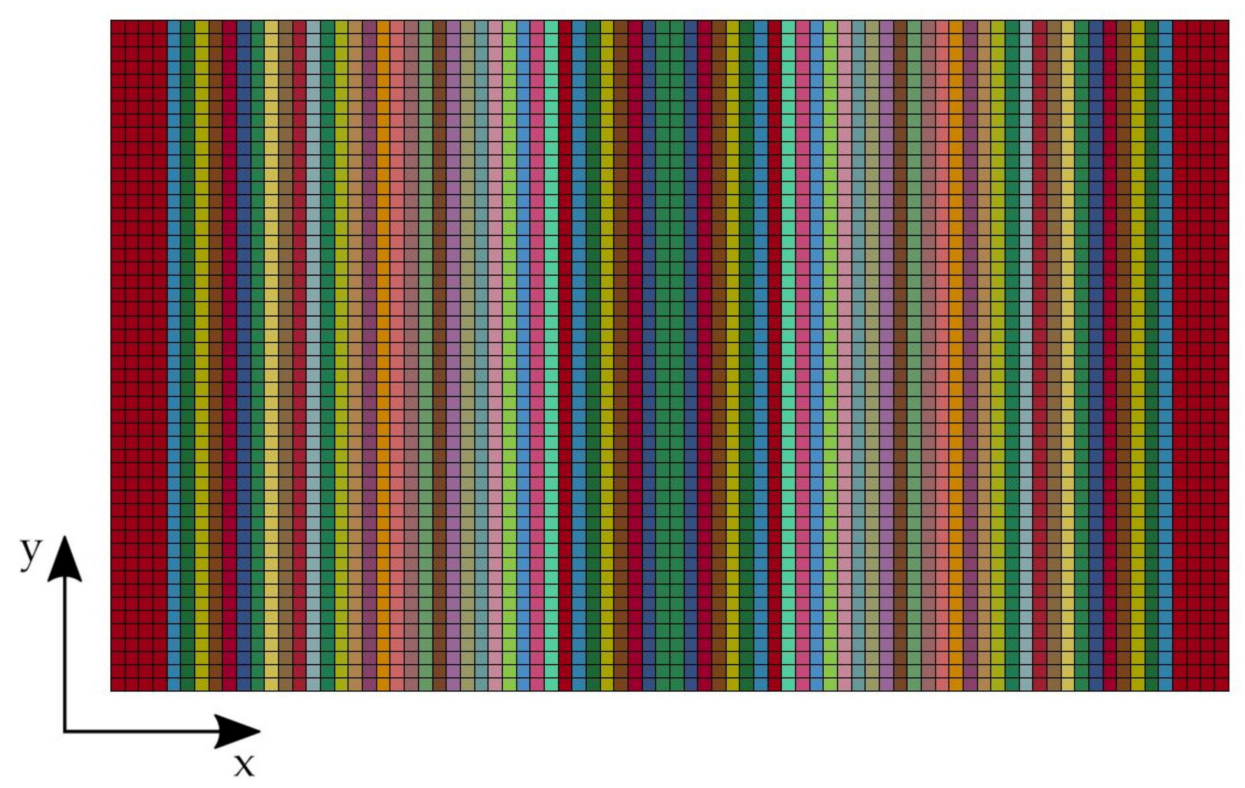

Figure 8. Top view of the finite element model of the composite laminate: longitudinal division of layers.

\subsection{Validation: Comparison of Experimental and Numerical Results}

The model of the composite plate with variable elastic modulus is adopted for simulating the four-point bending test. Results of the numerical force-displacement curve are then compared to those obtained with the progressively damaged plate. As shown in Figure 9, experimental and numerical results are in very good agreement, with limited discrepancies in slope. Results of numerical analysis are once again reported from $1 \mathrm{~mm}$ of displacement. The numerical displacement is measured on the rigid cylinders and these are initially distanced from the composite laminate, in order to avoid penetrations and numerical instabilities. Consequently, the initial path of the numerical force-displacement curve concerns the contact between laminate and cylinders and is not significant for the purpose.

Experimental response shows an increasing stiffness as the plate is bent. This hardening effect can be explained by taking into account that the considered material fails under compressive loads, as shown in Figure 4. The four-point bending tests are repeated until incipient failures occur. Consequently, cracks propagate in the upper layers subjected to compressive loads. When testing the composite plate, these cracks are progressively closed under compression. This allows to sustain loads in the failed layers and an increasing stiffness is thus obtained. The hardening effect can be also appreciated in the force-displacement curves with progressive damage of Figure 5 (dotted lines).

The limited discrepancy between experimental and numerical results highlights the accuracy of the $D I_{d}$ technique in mapping the residual elastic properties of the damaged plate. Further, this result validates the proposed approach which accounts for the variable elastic modulus with a continuous polynomial curve in the finite element model. 


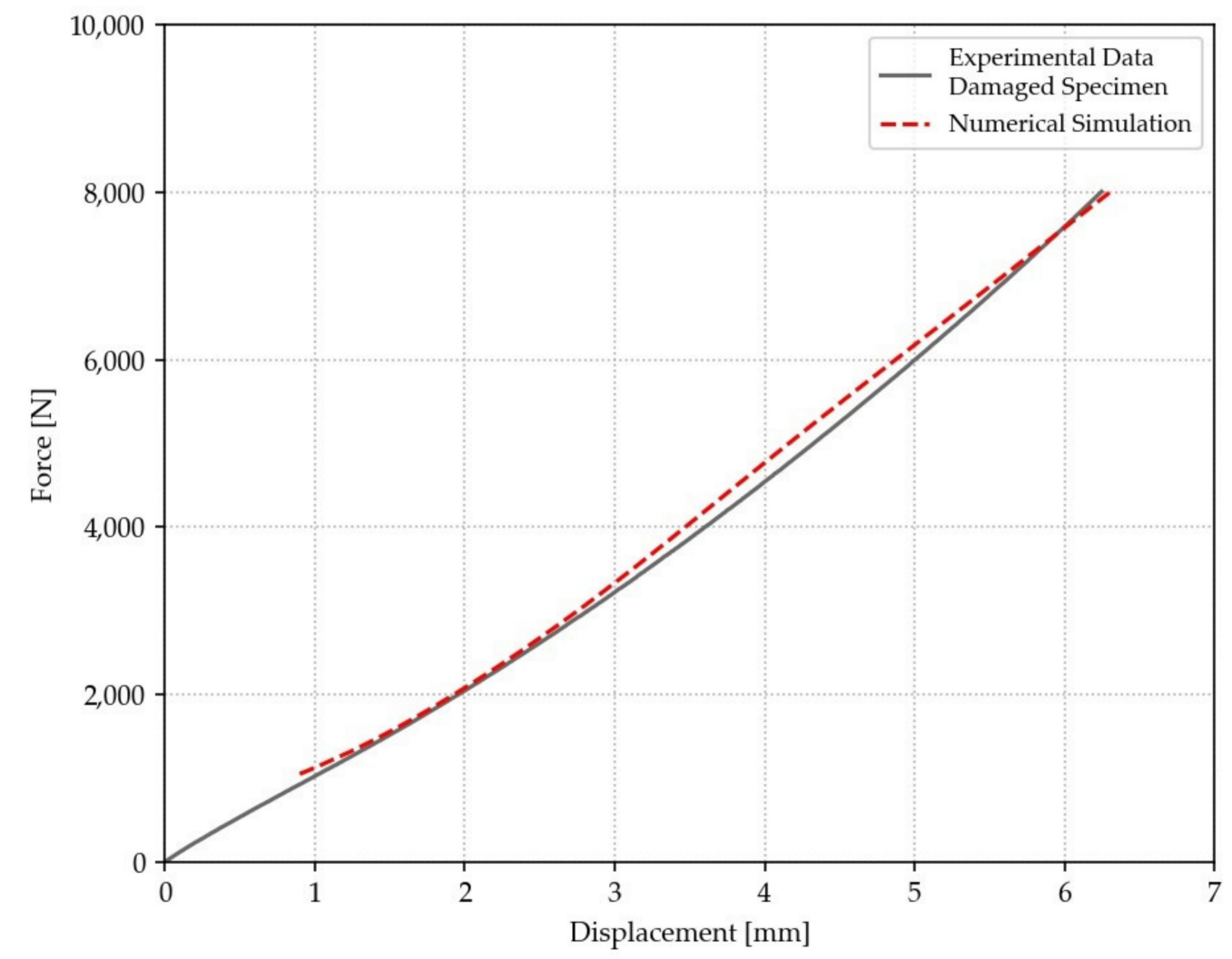

Figure 9. Comparison of experimental and numerical results of damaged specimen.

\section{Conclusions}

The use of the $D I_{d}$ technique in combination with the finite element method was presented. A four-point bending test was performed on an 8 layers twill fabric composite laminate until complete failure occurred. Experimental results were used to set up a finite element model of the composite plate subjected to bending test.

Then, repeated four-point bending tests allowed to progressively damage an undamaged plate of the same composite material. The $D I_{d}$ technique was adopted to map the residual elastic properties on the damaged plate. Seven locations have been considered along the middle line of the composite laminate. In particular, damage was mainly localized in correspondence of the inner noses. Further, it has been found that elastic properties were assessed as an average of the circular unclamped region considered for the $D I_{d}$ test, whose diameter is $76 \mathrm{~mm}$. The diameter of the analyzed region may thus be reduced in order to achieve very local assessments.

A continuous polynomial curve was then considered to account for the variable elastic modulus in the finite element model. The resulting force-displacement curve of the numerical analysis was in very good agreement with experimental data of damaged plate.

Therefore, the $D I_{d}$ methodology allows to locally assess the residual elastic properties of damaged composite materials. By mapping the elastic properties on the component and considering the assessed values in a finite element model, a precise description of the mechanical behavior of the composite plate is obtained.

Consequently, thanks to the proposed methodology, the health state of a damaged component can be quantitatively evaluated and decisions on its maintenance can be made by defining limits on the acceptable damage level. 
Author Contributions: Conceptualization, G.B.and D.P.; Data curation, C.N. and A.T.; Funding acquisition, C.N., A.T. and R.C.; Investigation, C.N., A.T. and R.C.; Methodology, G.B. and D.P.; Project administration, G.B. and D.P.; Software, C.N.; Supervision, G.B. and D.P.; Validation, C.N. and A.T.; Visualization, C.N.; Writing-original draft, C.N.; Writing-review and editing, A.T., R.C., G.B. and D.P.

Funding: This research received no external funding.

Conflicts of Interest: The authors declare no conflict of interest.

\section{References}

1. Heslehurst, R.B. Defects and Damage in Composite Materials and Structures; CRC Press: Boca Raton, FL, USA, 2017.

2. Ibrahim, M.E. Nondestructive testing and structural health monitoring of marine composite structures. In Marine Applications of Advanced Fibre-Reinforced Composites; Woodhead Publishing: Cambridge, UK, 2016; pp. 147-183.

3. Hubschen, G.; Altpeter, I.; Tschuncky, R.; Herrmann, H. Materials Characterization Using Nondestructive Evaluation (NDE) Methods; Woodhead Publishing: Cambridge, UK, 2016.

4. Katunin, A.; Danczak, M.; Kostka, P. Automated identification and classification of internal defect in composite structures using computed tomography and 3D wavelet analysis. Arch. Civ. Mech. Eng. 2015, 15, 436-448. [CrossRef]

5. Junyan, L.; Liqiang, L.; Yang, W. Experimental study on active infrared thermography as a NDI tool for carbon-carbon composites. Compos. Part B Eng. 2013, 45, 47-138. [CrossRef]

6. Tam, J.H.; Ong, Z.C.; Ismail, Z.; Ang, B.C.; Khoo, S.Y. Identification of material properties of composites materials using nondestructive vibrational evaluation approaches: A review. Mech. Adv. Mater. Struct. 2017, 24, 971-986. [CrossRef]

7. ASTM. E1876-15 Standard Test Method for Dynamic Young's Modulus, Shear Modulus, and Poisson's Ratio by Impulse Excitation of Vibration; ASTM International: West Conshohocken, PA, USA, 2015.

8. ASTM. C1259-15 Standard Test Method for Dynamic Young's Modulus, Shear Modulus, and Poisson's Ratio for Advanced Ceramics by Impulse Excitation of Vibration; ASTM International: West Conshohocken, PA, USA, 2015.

9. Paolino, D.S.; Geng, H.; Scattina, A.; Tridello, A.; Cavatorta, M.P.; Belingardi, G. Damaged composites laminates: Assessment of residual Young's modulus through the Impulse Excitation Technique. Compos. Part B Eng. 2017, 128, 76-82. [CrossRef]

10. Garnier, C.; Pastor, M.L.; Eyma, F.; Lorrain, B. The detection of aeronautical defects in situ on composite structures using Non-Destructive Testing. Compos. Struct. 2011, 93, 36-1328. [CrossRef]

11. Belingardi, G.; Cavatorta, M.P.; Paolino, D.S. A new damage index to monitor the range of the penetration process in thick laminates. Compos. Sci. Technol. 2008, 68, 2646-2652. [CrossRef]

12. Belingardi, G.; Cavatorta, M.P.; Paolino, D.S. Repeated impact response of hand layup and vacuum infusion thick glass reinforced laminates. Int. J. Impact Eng. 2008, 35, 609-619. [CrossRef]

13. Belingardi, G.; Cavatorta, M.P.; Paolino, D.S. On the rate of growth and extent of the steady damage accumulation phase in repeated impact tests. Compos. Sci. Technol. 2009, 69, 1693-1698. [CrossRef]

14. Belingardi, G.; Cavatorta, M.P.; Paolino, D.S. Single and repeated impact tests on fiber composite laminates: Damage index vs. residual flexural properties. In Proceedings of the 17th International Conference for Composite Materials. Edinburgh, UK, 27-31 July 2009.

15. Tridello, A.; D'Andrea, A.; Paolino, D.S.; Belingardi, G. A novel methodology for the assessment of the residual elastic properties in damaged composite components. Compos. Struct. 2017, 161, 435-440. [CrossRef]

16. Yam, L.H.; Wei, Z.; Cheng, L. Nondestructive detection of internal delamination by vibration-based method for composite plates. J. Compos. Mater. 2004, 38, 98-2183. [CrossRef]

17. LSTC. LS-DYNA Keyword Manual Volume I. 2019.

18. LSTC. LS-DYNA Keyword Manual Volume II. 2019.

19. Muflahi, S.A.; Mohamed, G.; Hallett, S.R. Investigation of delamination modeling capabilities for thin composite structures in LS-DYNA. In Proceedings of the 13th International LS-DYNA Users Conference, Detroit, MI, USA, 8-10 June 2014. 
20. Williams, K.V.; Vaziri, R.; Floyd, A.M.; Poursatip, A. Simulation of damage progression in laminated composite plates using LS-DYNA. In Proceedings of the 5th International LS-DYNA Users Conference, Southfield, Dearborn, MI, USA, 9-11 April 1998.

21. Moncayo, E.D.; Wagner, H.; Dreschler, K. Benchmarks for composite delamination using LS-DYNA 971: Low velocity impact. In Proceedings of the German LS-DYNA Forum, Frankenthal, Gemany, 11-12 October 2007.

22. ASTM. D6272-17. Standard Test Method for Flexural Properties of Unreinforced and Reinforced Plastics and Electrical Insulating Materials by Four-Point Bending; ASTM International: West Conshohocken, PA, USA, 2017.

23. ASTM. D5628-10. Standard Test Method for Impact Resistance of Flat, Rigid Plastic Specimens by Means of a Falling Dart (Tup or Falling Mass); ASTM International: West Conshohocken, PA, USA, 2010.

24. $\quad$ LSTC. LS-DYNA Theory Manual. 2019.

(C) 2019 by the authors. Licensee MDPI, Basel, Switzerland. This article is an open access article distributed under the terms and conditions of the Creative Commons Attribution (CC BY) license (http://creativecommons.org/licenses/by/4.0/). 